\title{
Sequencing of neurofilament genes identified NEFH Ser787Arg as a novel risk variant of sporadic amyotrophic lateral sclerosis in Chinese subjects
}

Feng Lin' ${ }^{1}$ Wanhui Lin ${ }^{1}$, Chaofeng Zhu ${ }^{1}$, Jilan Lin ${ }^{1}$, Junge Zhu², Xu-Ying Li ${ }^{2}$ Zhanjun Wang ${ }^{2}$, Chaodong Wang ${ }^{2}$ and Huapin Huang ${ }^{1 *}$

\begin{abstract}
Background: Amyotrophic lateral sclerosis (ALS) is a devastating neurodegenerative disease with neuronal cell inclusions composed of neurofilaments and other abnormal aggregative proteins as pathological hallmarks. Approximately $90 \%$ of patients have sporadic cases (SALS), and at least 4 genes, i.e. C9orf72, SOD1, FUS and TARDBP, have been identified as the main causative genes, while many others have been proposed as potential risk genes. However, these mutations could explain only $~ 10 \%$ of sALS cases. The neurofilament polypeptides encoded by NEFH, NEFM, and NEFL are promising protein biomarkers for ALS and other degenerative diseases. However, whether the genetic variants of these genes were associated with ALS remain ambiguous.
\end{abstract}

Methods: Here, we used PCR-Sanger to sequence the exons of these three genes in a cohort of 371 sALS patients and 711 healthy controls (Phase I) and validated the risk variant in another 300 sALS patients and 1076 controls (Phase II).

Results: A total of 92 variants were identified, including 36 rare heterozygous variants in NEFH, 27 in NEFM, and 16 in NEFL, and only rs568759161 (p.Ser787Arg) in NEFH reached nominal statistical power ( $P=0.02$ at Phase l, $P=0.009$ at Phase II) in the case-control comparison. Together, the Phase I and II studies showed the significantly higher frequency of the variant in cases $(9 / 1342,0.67 \%)$ than in controls $(2 / 3574,0.07 \%)(O R$ 12.06; 95\% Cl 2.60-55.88; $P=0.0003)$. No variants passed multiple testing in the discovery cohort, but rs568759161 was associated with ALS in a replication cohort.

Conclusions: Our results confirmed that NEFH Ser787Arg is a novel sALS risk variant in Chinese subjects, but NEFM and NEFL were not associated with SALS. These data may have implications for genetic counselling and for understanding the pathogenesis of SALS.

Keywords: Sporadic amyotrophic lateral sclerosis, Neurofilament genes, Rare variant, Association

*Correspondence: hh-p@163.com

1 Department of Neurology, Fujian Medical University Union Hospital, Fujian 350001, China

Full list of author information is available at the end of the article

\section{Introduction}

Amyotrophic lateral sclerosis (ALS) is a devastating neurodegenerative disease characterized by loss of motor neurons in the brain and spinal cord, resulting in muscle atrophy, swallowing disorders, and pyramidal tract signs. A known pathological hallmark of ALS is neuronal cell 
inclusions composed of neurofilaments and other abnormal aggregative proteins [1-3]. Epidemiological surveys show an incidence of $0.6-3.8$ per 100,000 persons per year and a prevalence of 4.1-8.4 per 100,000 persons worldwide [4]. It has been reported that the yearly incidence is 0.8 (2010-2015) per 100,000 persons in China [5]. Approximately $10 \%$ of cases were familial, and $90 \%$ were sporadic cases. To date, the genetics of ALS are not fully understood. In 1993, SOD1 was discovered as the first ALS-causing gene. Since then, many other genes have been reported to be causative for (i.e. C9orf72, SOD1, FUS, TARDBP, etc.) or associated with the disease $[6,7]$. Genetic studies have found that mutations in these genes were mainly identified in familial cases and could explain only approximately $10 \%$ of sporadic cases (sALS) [6]. With next-generation sequencing, novel genes and loci have been increasingly discovered [8], but the genetics of sALS are not fully understood.

Neurofilaments (NFs) are type IV intermediate filament heteropolymers composed of light (NEFL), medium (NEFM), and heavy (NEFH) subunits. The different NF subunits have the same conserved alpha-helical rod domain and differ in the head and tail domains. NFs function by determining axonal calibre, promoting axonal growth, and forming a 3-dimensional lattice that supports cytoplasmic organelle organization [9]. NFs have been considered to play an essential role in many neurodegenerative diseases, such as ALS, CharcotMarie-Tooth disease, and Parkinson's disease [10]. Many studies have revealed the relationship between NFs and ALS. First, one of the important pathological features of ALS is the cytoplasmic inclusion bodies of NFs [11-14]. Second, motor function impairment is observed in NFsubunit-transgenic mice $[15,16]$. Third, studies have found a decrease in NEFL mRNA expression in the spinal cord tissue of patients with ALS $[17,18]$. Recently, NEFL and phosphorylated NEFH (pNEFH) were considered as promising novel biomarkers in the blood and cerebrospinal fluid of ALS patients during disease onset and progression [19-21]. In previous studies, NEFH variants were reported in approximately $1 \%$ sALS cases [22-26], and $N E F H$ was considered a susceptibility gene for ALS. However, the conclusions from many different studies are contradictory [27-29]. The mechanism may involve abnormal protein modification, folding, clearance, and axonal transport [30]. However, NEFM and NEFL have not been linked to ALS, although upregulation of NEFM has been detected in spinal cord tissues in patients with ALS or ALS-like diseases [31, 32]. Thus far, systemic sequencing studies of NEFH/NEFM/NEFL with large samples have been rare, and most of the relevant studies have been restricted to the Caucasian population. Moreover, the distribution, burden, and significance of these genetic variations remain ambiguous, especially in the Chinese sALS cohort.

In this study, we sequenced the variants in the exons of NEFH, NEFM, and NEFL in a Chinese sALS cohort including 371 sALS patients and 711 healthy controls (Phase I) to identify the potentially associated variants and validate these variants in another 300 sALS patients and 1076 controls (Phase II). We found that rs568759161 (p.Ser787Arg) in NEFH was a novel risk variant associated with sALS, and the distribution of this genetic variant was different from that observed in previous studies. However, NEFM and NEFL were not definitively associated with SALS.

\section{Materials and methods}

\section{Patients and controls}

A total of 371 sALS patients and 711 healthy subjects of Han ethnicity were recruited from the Department of Neurology of three hospitals (Fujian Medical University Union Hospital, Sanming First Hospital Affiliated to Fujian Medical University, and Xuanwu Hospital of Capital Medical University) from Jan 2016 to Nov 2020 (Phase I). Another 1076 healthy elderly control subjects were recruited from communities in Beijing as a further validation control group, and 300 sALS patients were recruited from the aforementioned hospitals to confirm the risk variant (Phase II). The inclusion criteria of the control group were healthy elderly people without diseases history of motor neuron diseases, degenerative neurological disorders or malignancy.

All patients with ALS were diagnosed by at least two neuromuscular specialists in each hospital based on clinical and electrophysiological findings according to the revised El Escorial criteria [33]. We only recruited sporadic cases in the study, which were defined as the absence of a second patient within three generations of the family, and frontotemporal dementia (FTD) was excluded in each patient in this study. And all sALS patients fulfilled the criteria for probable, or definite ALS based on this criterion. Clinical data, including age at onset (AAO), initial site of impairment, core symptoms and signs, electromyography, and nerve conduction velocity assessment, were reviewed and analysed reciprocally by researchers and specialists from the hospitals. Family inquiry was performed to exclude the existence of kinship among samples within at least three generations.

\section{NEFH/NEFM/NEFL genetic analysis}

Three millilitres of blood were obtained from sALS patients and controls. Polymerase chain reaction (PCR) assays and extension primers for exons were designed using Oligo 6.0 software (Molecular Biology Insights, Inc., CO, USA). The primer sequences for amplifying 
the exons of NEFL, NEFM, and NEFH are listed in Additional file 1: Table S1. PCR products were purified and sequenced by an ABI 3730 DNA Analyzer (Applied Biosciences, Inc., CT, USA). Chromas 2.22 software was used for sequence reading. The variant position at the genomic level was based on GenBank accession number NC_000022.10/NC_000008.10/NC_000008.10, the transcript position was based on NM_021076.3/ NM_005382.2/NM_006158.4, and the protein-level position was based on NP_066554.2/NP_005373.2/ NP_006149.2, according to the hg19/GRCh37 reference sequence. The chromosomal position, frequencies, and other relevant information of the variants were annotated using the 1000 Genome Project database (http://www.1000genomes.org), dbSNP version 147 (http://www.ncbi.nlm.nih.gov/projects/SNP), and the Exome Aggregation Consortium (ExAC) (http:// exac.broadinstitute.org/) database. Variants were classified into the following categories according to their minor allele frequencies (MAFs): MAF $>0.05$, common variant; $0.01 \leq \mathrm{MAF} \leq 0.05$, low-frequency variant; and $\mathrm{MAF}<0.01$, rare variant. We paid close attention to the rare variant $(\mathrm{MAF}<0.01)$. The ExAC_EAS (for the East Asian population) and GnomAD_exome_EAS databases were used as references. Novel variants were defined as those that were not indexed in any of the databases, irrespective of ethnic population. The functional effect of the variants was predicted by combined annotation-dependent depletion (CADD) (https://cadd.gs.washington.edu/ snv).

\section{Statistical analysis}

Low-frequency and common variants located in the $N E F H, N E F M$, and NEFL coding regions in the control group were tested for deviations from Hardy-Weinberg equilibrium using the $\chi^{2}$ test. Allele frequencies of common and low-frequency variants in patients and controls were compared by $x^{2}$ statistics using SPSS 22 software. Nominal $P$ values were corrected for the number of variants tested using Bonferroni correction. The burden test for rare coding variants across the full $N E F H, N E F M$, and
NEFL coding sequences was performed by the sequence kernel association test (SKAT-O) using $\mathrm{R}$ software (version 4.0.0). Differences in AAO between patients carrying and not carrying rare variants were calculated using an unpaired nonparametric (Mann-Whitney) test. $P<0.05$ was considered statistically significant.

\section{Results \\ Demographic data of the cohort}

The cohort consisted of two phases of cases and controls. Phase I included 371 patients with sALS and 711 healthy controls. The AAO of the patients was $53.42 \pm 10.28$ years. Phase II included another 300 sALS patients and 1076 controls. The AAO was $53.49 \pm 9.56$ years in the cases. It has been reported that ALS was more prevalent in men, and the mean AAO was 51 (IQR 43-59) years in China [34]. Therefore, we chose more female individuals in the controls, who were older $(69.41 \pm 8.42$ and $69.83 \pm 7.70$ years) than the cases selected (Table 1).

\section{Rare coding variants identified in the NEFH, NEFM, and NEFL genes}

We screened the exons and their flanking sequences in the NEFH, NEFM, and NEFL genes by PCR and Sanger sequencing (Additional file 6: Fig. S1). We identified 92 variants, including 36 rare heterozygous variants in $N E F H, 27$ in NEFM, and 16 in NEFL. There were 16 rare coding variants of NEFH in 20 sALS cases, 8 of NEFM in 9 sALS cases, and 10 of NEFL in 17 sALS cases. The rare variants included 1 stop-gain, 1 stop-loss, 4 frameshift, 2 insertion/deletion, 44 missense, 1 intron-harboured, and 26 synonymous variants in 44 sALS cases ( 20 cases carried rare variants in NEFH, 9 in NEFM, and 17 in NEFL). Of the sALS patients who carried rare variants, 2 had variants in two genes. Of the rare nonsynonymous variants, 4 in NEFH, 2 in NEFM, and 3 in NEFL were identified only in cases, while 14 in NEFH, 13 in NEFM, and 5 in NEFL were identified only in control subjects. Seven variants in NEFH, 3 in NEFM, and 2 in NEFL were identified in both the cases and controls. Given these genes,

Table 1 Demographic data of the study subjects

\begin{tabular}{|c|c|c|c|c|c|c|}
\hline \multirow[t]{2}{*}{ Clinical features } & \multicolumn{2}{|l|}{ Phase I } & \multicolumn{2}{|l|}{ Phase II } & \multicolumn{2}{|l|}{ Combined } \\
\hline & $\operatorname{sALS}(n=371)$ & Control $(n=711)$ & $\operatorname{sALS}(n=300)$ & Control $(n=1076)$ & $\operatorname{sALS}(n=671)$ & Control_2 $(n=1787)$ \\
\hline Sex, M/F (ratio) & $227 / 144(1.58: 1)$ & 283/428 (0.66:1) & $183 / 117(1.56: 1)$ & 453/623 (0.73:1) & $410 / 261(1.57: 1)$ & 736/1051 (0.70:1) \\
\hline Age (year, mean $\pm S D$ ) & $55.13 \pm 10.28$ & $69.41 \pm 8.42$ & $55.16 \pm 9.82$ & $69.83 \pm 7.70$ & $55.14 \pm 9.97$ & $69.66 \pm 7.91$ \\
\hline $\begin{array}{l}\text { Age at onset (year, } \\
\text { mean } \pm S D)\end{array}$ & $53.42 \pm 10.28$ & - & $53.49 \pm 9.56$ & - & $53.45 \pm 9.96$ & - \\
\hline Site of onset, bulbar (\%) & 73/371 (19.68\%) & - & $59 / 300(19.61)$ & - & 132/671 (19.67\%) & - \\
\hline
\end{tabular}

sALS, sporadic amyotrophic lateral sclerosis; AAO, age at onset 
9 heterozygous missense mutations, 1 in-frame deletion, and 1 nonsense mutation in NEFH were distributed in 4 exons, especially in exons 4 and 1; 5 missense variants were distributed in exons 3 and 1 in NEFM; and 1 stoploss, 1 in-frame deletion and 3 missense variants were distributed in the exons of NEFL (Fig. 1). Upon comparing the cases and controls, we found that only one variant, rs568759161 (c.2361C > G, p.Ser787Arg), in NEFH was nominally more frequent in cases than in controls (OR 9.64; 95\% CI 1.12-82.67; $P=0.02$ ) (Table 2 and Additional file 2: Table S2). However, no variants passed the Bonferroni multiple comparison test.

\section{Burden test for rare variants of NEFH and NEFL in ALS}

To investigate the enrichment of rare coding variants in ALS, we performed the SKAT-O burden test for each gene. We chose the dominant inheritance (Dom) model for nonsynonymous coding variants and the non-benign and loss-of-function (LoF) variants. As shown in Additional file 3: Table S3, none of the genes showed significant enrichment of rare variants in cases.

\section{Validation of the association of NEFH Ser787Arg variant with sALS}

No variants passed multiple testing in the discovery cohort, in which the only rare variant, rs568759161 (p.Ser787Arg), in NEFH, was nominally associated with ALS. This variant was found in 5 sALS patients and 1 control in Phase I (MAF: 5/742, 0.67\% and 1/1422, 0.07\%; $P=0.02$; OR 9.64; 95\% CI 1.12-82.67). Due to the lack of significance in the Bonferroni correction, we added 300 cases and 1076 controls for sequencing (Phase II validation). The variant was found in 4 sALS patients and 1 control in Phase II (MAF: 4/600, 0.67\% and 1/2152, $0.05 \% ; P=0.009$; OR 14.43; 95\% CI 1.61-129.40). Upon combining the two phases, the variant was shown to be significantly more abundant in cases than in controls (OR 12.06; 95\% CI 2.60-55.88; $P=0.0003$ ) (Table 3).

In clinical aspects, one female and 8 male sALS patients shared the NEFH p.Ser787Arg variant with AAO at $53.44 \pm 13.51$ years, which was not different from other sALS cases. Most (7/9) of these patients initially presented with limb symptoms (Additional file 4: Table S4).

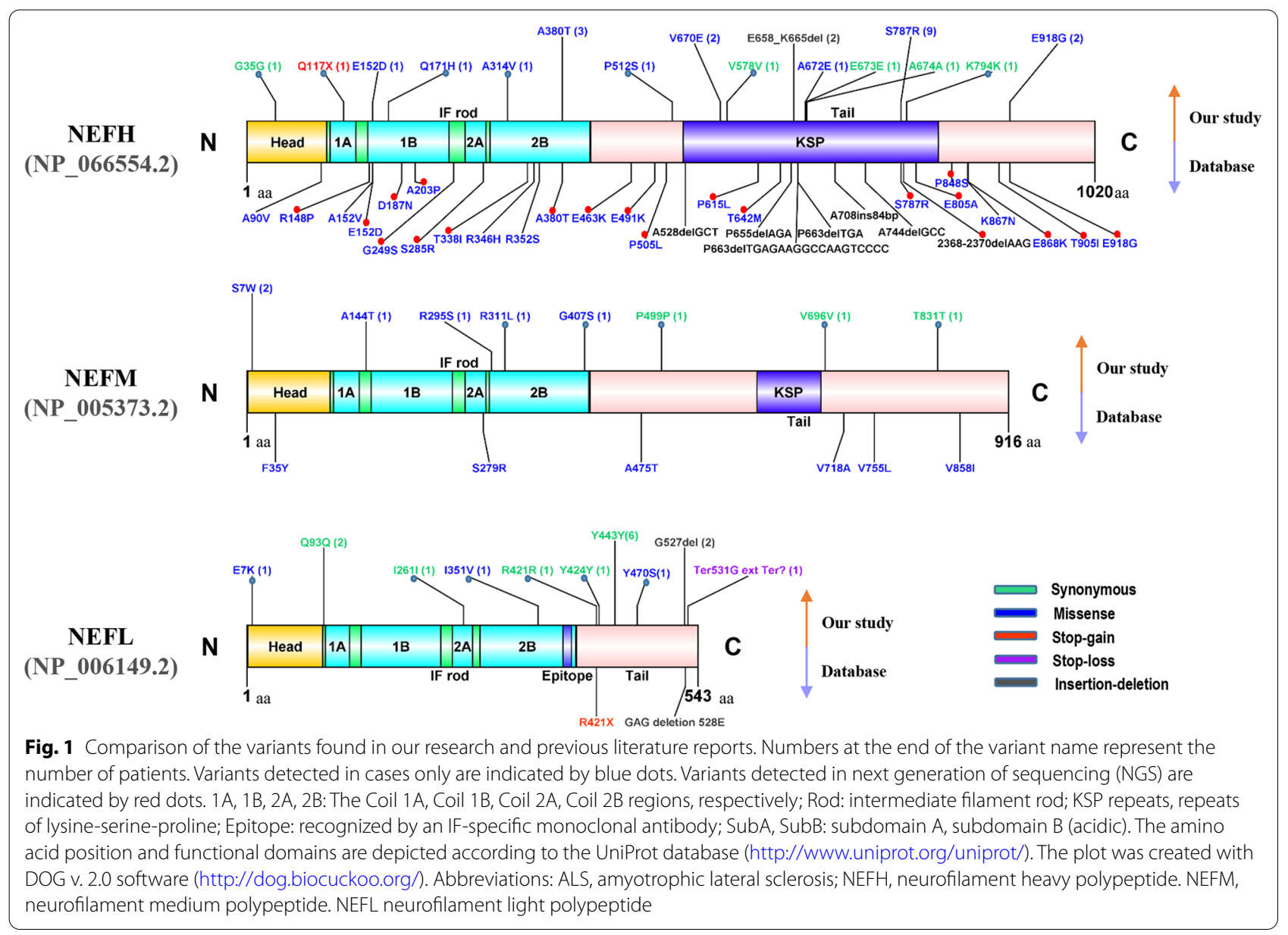




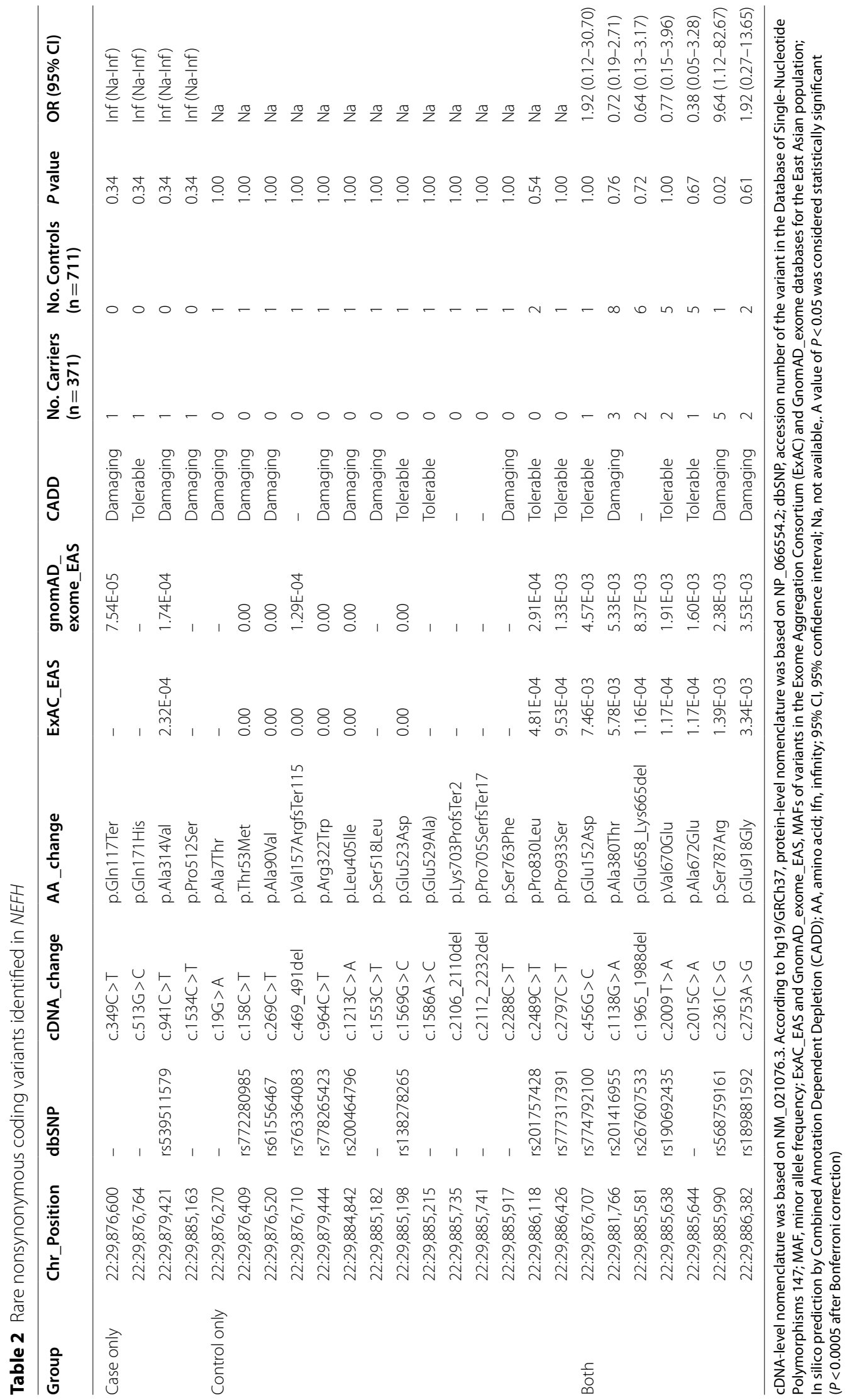


Table 3 Statistical outcome in variant (NEFH p.Ser787Arg) carriers

\begin{tabular}{|c|c|c|c|c|c|c|}
\hline \multirow[t]{2}{*}{ Variables } & \multicolumn{2}{|l|}{ Phase I } & \multicolumn{2}{|l|}{ Phase II } & \multicolumn{2}{|l|}{ Combined } \\
\hline & $\operatorname{sALS}(n=371)$ & Control $(n=711)$ & $\operatorname{sALS}(n=300)$ & Control $(n=1076)$ & $\operatorname{sALS}(n=671)$ & Control_2 $(n=1787)$ \\
\hline No. carriers & 5 & 1 & 4 & 1 & 9 & 2 \\
\hline MAF & $6.73 \mathrm{E}-03$ & 7.03 E-04 & $6.67 \mathrm{E}-03$ & $4.65 \mathrm{E}-04$ & $6.71 \mathrm{E}-03$ & $5.60 \mathrm{E}-04$ \\
\hline$P$ value & 0.02 & & 0.009 & & 0.0003 & \\
\hline OR $(95 \% \mathrm{Cl})$ & $9.64(1.12-82.67)$ & & $14.43(1.61-129.40)$ & & $12.06(2.60-55.88)$ & \\
\hline
\end{tabular}

SALS, sporadic amyotrophic lateral sclerosis; MAF, minor allele frequency; $P$ value, determined using Fisher's exact test; $95 \% \mathrm{Cl}, 95 \%$ confidence interval

\section{Low-frequency and common coding variants identified in the NEFH, NEFM, and NEFL genes}

Among all the nonsynonymous variants, 5 low-frequency $(0.01 \leq \mathrm{MAF} \leq 0.05)$ were identified in $\mathrm{NEFH}, 1$ was in NEFM and 1 was in NEFL. For common (MAF>0.05) variants, 4 were revealed in NEFH, 1 was in NEFM and 1 was in NEFL (Additional file 5: Table S5). The results suggested that genotype frequencies of NEFH, NEFM and $N E F L$ were Hardy-Weinberg equilibrium in the control group. However, there were no statistically significant differences in common or low-frequency variants between the case and control groups.

\section{Discussion}

In our study, it was found that many domains harboured rare variants in NEFH, NEFM, and NEFL in sALS patients, which is different from the results of previous studies [35] and the ALSod database (https://alsod.ac. uk/output/gene.php\#variants). Previous ALS studies have indicated that the NEFH mutations are insertions and deletions and are mostly located at the tail $[22,26]$. Our study demonstrated more point variants than insertion/deletion variants in NEFH (Fig. 1). Second, our data showed more carriers with rare nonsynonymous variants (17/371, 4.58\%) in NEFH than previous reports showed in other populations [25, 29, 36-38]. Third, mutation of NEFL has traditionally been recognized as a cause of Charcot-Marie-Tooth disease [39], congenital myopathy in humans [40], and motor neuron disease in mice [32]. NEFM is linked to Parkinson's disease [41]. However, $N E F L$ and NEFM were not associated with sALS in our study. Although we had shown variants in these genes in sALS, we did not find significant differences in clinical characteristics (sex, AAO, onset site) between cases carrying and not carrying the variants. The differences further confirmed the genetic heterogeneity in sALS among different ethnicities and highlight the association of NEFH, but not NEFL or NEFM, with ALS.

This study found that the p.Ser787Arg variant in $N E F H$ was associated with sALS in Chinese subjects. Notably, rs568759161 is only found in only the East
Asian population according to ExAC (MAF 0.14\%) and gnomAD (MAF 0.24\%), and their MAFs were slightly higher than those of our control group $(0.07 \%$ in Phase I and $0.05 \%$ in Phase II) (Table 3). We assume that the difference in allele frequency between the two databases and our study might be due to the population differences. Moreover, we found that some variants reported by other studies were not associated with ALS. For example, A380T in NEFH was identified only in cases previously [29], but our study suggested it was identified in both case and control groups. So, we believed study of rare variants need large samples of controls. In our study, we recruited relatively large controls ( $n=711$ in Phase I and $n=1076$ in Phase II) to decrease the chance of false positive or false negative.

The phosphorylation of NF subunits has been considered a critical process regulating the formation and function of NFs [10]. The variant p.Ser787Arg is located in the phosphorylated region in a conserved sequence. Proper phosphorylation/dephosphorylation of NEFH may be considered a protective mechanism under conditions of cellular stress [16, 42], indicating that the modification of NEFH plays a significant role in maintaining the normal function of neurons. We hypothesized that the NEFH-S787R variant changes the phosphorylation of the protein. However, because of the unavailability of an antibody against the site, we did not test the hypothesis in this study. In the future, we need to synthesize antibodies against the phosphorylated NEFH-Ser787 site to further explore the changes in phosphorylation levels.

Recently, next-generation sequencing technology have identified many genes, including NEFH [43], as causative for or associated with ALS. In NEFH, 20 variants have been reported in ALS cases (Fig. 1) [25, $36-38,44,45]$, but none was conclusively related to the disease. The p.Ser787Arg was only reported by Chen et al. [45], but its association with ALS was not confirmed. Our study provided the spectrum of NEFH variants and confirmed the association of p. Ser787Arg with Chinese sALS. 


\section{Conclusion}

In this study, we analysed the mutational spectrum of $N E F H, N E F M$, and NEFL genes in an sALS Chinese cohort and identified the variant (rs568759161) locating in the phosphorylated site of the KSP domain of $N E F H$ as a risk variant associated with sALS in Chinese. Functional studies will be necessary to assess its role in ALS pathogenesis.

\section{Abbreviations}

ALS: Amyotrophic lateral sclerosis; NFs: Neurofilaments; FTD: Frontotemporal dementia; AAO: Age at onset; PCR: Polymerase chain reaction; ExAC: Exome aggregation consortium; MAFs: Minor allele frequencies; CADD: Combined annotation-dependent depletion; SKAT-O: Sequence kernel association test; LoF: Loss-of-function.

\section{Supplementary Information}

The online version contains supplementary material available at https://doi. org/10.1186/s12920-021-01073-z.

Additional file 1. Primers for amplification of exons.

Additional file 2. Rare non-synonymous coding variants in NEFM and NEFL.

Additional file 3. Burden in all rare variants.

Additional file 4. Clinical features of NEFH (S787R) carriers.

Additional file 5. Low frequency and common variants identified in NEFH, NEFM and NEFL genes.

Additional file 6 . Workflow of the study design

\section{Acknowledgements}

We thank all the participants of this study. We're also grateful for Dr. Xiuli Feng and Dr. Shu Xie for their technical assistance, including PRC and Sanger sequencing.

\section{Authors' contributions}

$\mathrm{FL}$ and $\mathrm{HH}$ initiated the project and wrote the manuscript. $\mathrm{FL}, \mathrm{WL}$, and $\mathrm{CZ}$ designed the experiments. FL, CZ, JL, and XL performed the experiments. $\mathrm{FL}, \mathrm{ZW}$, and $\mathrm{CW}$ analysed the data. $\mathrm{FL}, \mathrm{XL}, \mathrm{JZ}$ and $\mathrm{JL}$ constructed the figures. $\mathrm{FL}, \mathrm{XL}, \mathrm{JZ}$ and $\mathrm{XL}$ created the tables. All authors read and approved the final manuscript.

\section{Funding}

This study was supported by funds from the Innovation of Science and Technology, Fujian Province (Grant Number 2017Y9058), to Prof. Huapin Huang; the Fujian Provincial Health Technology Project (Grant Number 2019-ZQN-38), to Dr. Wanhui Lin; the Fujian Sanming Science and Technology Plan Project (Grant Number 2019-S-3) and Fujian Provincial Science and Technology Project (Grant Number 2020J011271), to Dr. Feng Lin; and the Ministry of Science and Technology (Grant Number 2016YFC1306000), Special Fund from Key Laboratory of Neurodegenerative Diseases, Ministry of Education of China (PXM2019_026283_000002), and National Natural Science Foundation (Grant Number 81771212) to Prof. Chaodong Wang.

\section{Availability of data and materials}

All genetic polymorphisms identified in this study is available at Table 2, Additional file 2: Table S2 and Additional file 5: Table S5. The primer sequences are shown in Additional file 1: Table S1. The original sequencing and clinical datasets generated during the current study are not publicly available due to maintaining patient confidentiality but are available from the corresponding author (hh-p@163.com) on reasonable request.

\section{Declarations}

Ethics approval and consent to participate

All research participants or their legal representatives signed informed consent forms for participation in clinical and genetic research. The medical ethics committee approved the protocol and provided informed consent (2021KY016) of the whole study at Fujian Medical University Union Hospital, Fuzhou, China. In this study, all methods were performed in accordance with the relevant guidelines and regulations in accordance with the Declaration of Helsinki.

\section{Consent for publication}

Not applicable.

\section{Competing interests}

None of the authors declared conflict of interest.

\section{Author details}

${ }^{1}$ Department of Neurology, Fujian Medical University Union Hospital, Fujian 350001, China. ${ }^{2}$ Department of Neurology, Xuanwu Hospital of Capital Medical University, Beijing 100053, China.

Received: 7 June 2021 Accepted: 1 September 2021

Published online: 11 September 2021

\section{References}

1. Leung CL, He CZ, Kaufmann P, Chin SS, Naini A, Liem RK, et al. A pathogenic peripherin gene mutation in a patient with amyotrophic lateral sclerosis. Brain Pathol. 2004;14:290-6.

2. MCAlary L, Plotkin SS, Yerbury JJ, Cashman NR. Prion-like propagation of protein misfolding and aggregation in amyotrophic lateral sclerosis. Front Mol Neurosci. 2019;12:262.

3. Lin H, Schlaepfer WW. Role of neurofilament aggregation in motor neuron disease. Ann Neurol. 2006;60:399-406.

4. Longinetti E, Fang F. Epidemiology of amyotrophic lateral sclerosis: an update of recent literature. Curr Opin Neurol. 2019;32:771-6.

5. Zhou S, Zhou Y, Qian S, Chang W, Wang L, Fan D. Amyotrophic lateral sclerosis in Beijing: epidemiologic features and prognosis from 2010 to 2015. Brain Behav. 2018;8:e01131.

6. Taylor JP, Brown RH Jr, Cleveland DW. Decoding ALS: from genes to mechanism. Nature. 2016:539:197-206.

7. Hardiman O, Al-Chalabi A, Chio A, Corr EM, Logroscino G, Robberecht W, et al. Amyotrophic lateral sclerosis. Nat Rev Dis Primers. 2017;3:17085

8. Pampalakis G, Mitropoulos K, Xiromerisiou G, Dardiotis E, Deretzi G, Anagnostouli M, Katsila T, Rentzos M, Patrinos GP. New molecular diagnostic trends and biomarkers for amyotrophic lateral sclerosis. Hum Mutat. 2019;40(4):361-73.

9. Gentil BJ, Tibshirani M, Durham HD. Neurofilament dynamics and involvement in neurological disorders. Cell Tissue Res. 2015;360:609-20.

10. Al-Chalabi A, Miller CC. Neurofilaments and neurological disease. BioEssays. 2003;25:346-55.

11. Kobayakawa Y, Sakumi K, Kajitani K, Kadoya T, Horie H, Kira J, et al. Galectin-1 deficiency improves axonal swelling of motor neurones in SOD1(G93A) transgenic mice. Neuropathol Appl Neurobiol. 2015:41:227-44.

12. Hirano A, Donnenfeld $H$, Sasaki $S$, Nakano I. Fine structural observations of neurofilamentous changes in amyotrophic lateral sclerosis. J Neuropathol Exp Neurol. 1984;43:461-70.

13. Hirano A, Nakano I, Kurland LT, Mulder DW, Holley PW, Saccomanno G. Fine structural study of neurofibrillary changes in a family with amyotrophic lateral sclerosis. J Neuropathol Exp Neurol. 1984;43:471-80.

14. Sasaki S, Maruyama S, Yamane K, Sakuma H, Takeishi M. Swellings of proximal axons in a case of motor neuron disease. Ann Neurol. 1989;25:520-2.

15. Elder GA, Friedrich VL Jr, Pereira D, Tu PH, Zhang B, et al. Mice with disrupted midsized and heavy neurofilament genes lack axonal neurofilaments but have unaltered numbers of axonal microtubules. J Neurosci Res. 1999;57:23-32. 
16. Liu Q, Xie F, Siedlak SL, Nunomura A, Honda K, Moreira Pl, et al. Neurofilament proteins in neurodegenerative diseases. Cell Mol Life Sci. 2004;61:3057-75.

17. GeWW, Leystra-Lantz C, Wen W, Strong MJ. Selective loss of trans-acting instability determinants of neurofilament mRNA in amyotrophic lateral sclerosis spinal cord. J Biol Chem. 2003;278:26558-63.

18. Bergeron C, Beric-Maskarel K, Muntasser S, Weyer L, Somerville MJ, Percy ME. Neurofilament light and polyadenylated mRNA levels are decreased in amyotrophic lateral sclerosis motor neurons. J Neuropathol Exp Neurol. 1994;53:221-30

19. Poesen K, Van Damme P. Diagnostic and prognostic performance of neurofilaments in ALS. Front Neurol. 2018;9:1167.

20. Khalil M, Teunissen CE, Otto M, Piehl F, Sormani MP, Gattringer T, et al. Neurofilaments as biomarkers in neurological disorders. Nat Rev Neurol. 2018;14:577-89

21. Oeckl P, Jardel C, Salachas F, Lamari F, Andersen PM, Bowser R, et al. Multicenter validation of CSF neurofilaments as diagnostic biomarkers for ALS. Amyotroph Lateral Scler Frontotemporal Degener. 2016:17:404-13.

22. Figlewicz DA, Krizus A, Martinoli MG, Meininger V, Dib M, Rouleau GA, et al. Variants of the heavy neurofilament subunit are associated with the development of amyotrophic lateral sclerosis. Hum Mol Genet. 1994;3:1757-61.

23. Skvortsova V, Shadrina M, Slominsky P, Levitsky G, Kondratieva E, Zherebtsova A, et al. Analysis of heavy neurofilament subunit gene polymorphism in Russian patients with sporadic motor neuron disease (MND). Eur J Hum Genet. 2004;12:241-4.

24. Al-Chalabi A, Andersen PM, Nilsson P, Chioza B, Andersson JL, Russ C, et al. Deletions of the heavy neurofilament subunit tail in amyotrophic lateral sclerosis. Hum Mol Genet. 1999;8:157-64.

25. Tripolszki K, Gampawar P, Schmidt H, Nagy ZF, Nagy D, Klivényi P, et al. Comprehensive genetic analysis of a Hungarian amyotrophic lateral sclerosis cohort. Front Genet. 2019;10:732.

26. Tomkins J, Usher P, Slade JY, Ince PG, Curtis A, Bushby K, et al. Novel insertion in the KSP region of the neurofilament heavy gene in amyotrophic lateral sclerosis (ALS). NeuroReport. 1998;9:3967-70.

27. Vechio JD, Bruijn LI, Xu Z, Brown RH Jr, Cleveland DW. Sequence variants in human neurofilament proteins: absence of linkage to familial amyotrophic lateral sclerosis. Ann Neurol. 1996;40:603-10.

28. Rooke K, Figlewicz DA, Han FY, Rouleau GA. Analysis of the KSP repeat of the neurofilament heavy subunit in familiar amyotrophic lateral sclerosis. Neurology. 1996;46:789-90.

29. Garcia ML, Singleton AB, Hernandez D, Ward CM, Evey C, Sapp PA, et al. Mutations in neurofilament genes are not a significant primary cause of non-SOD1-mediated amyotrophic lateral sclerosis. Neurobiol Dis. 2006;21:102-9.

30. Julien JP. Amyotrophic lateral sclerosis. Unfolding the toxicity of the misfolded. Cell. 2001;104:581-91.

31. Wu YY, Kuo HC. Functional roles and networks of non-coding RNAs in the pathogenesis of neurodegenerative diseases. J Biomed Sci. 2020;27:49.

32. Bomont $\mathrm{P}$, Cavalier L, Blondeau F, Ben Hamida C, Belal S, Tazir M, et al. The gene encoding gigaxonin, a new member of the cytoskeletal BTB/ kelch repeat family, is mutated in giant axonal neuropathy. Nat Genet. 2000;26:370-4.

33. Brooks BR, Miller RG, Swash M, Munsat TL. World federation of neurology research group on motor neuron diseases: El Escorial revisited: revised criteria for the diagnosis of amyotrophic lateral sclerosis. Amyotroph Lateral Scler Other Motor Neuron Disord. 2000;1:293-9.

34. Dorst J, Chen L, Rosenbohm A, Dreyhaupt J, Hübers A, Schuster J, et al. Prognostic factors in ALS: a comparison between Germany and China. J Neurol. 2019;266:1516-25.

35. Yuan A, Rao MV, Veeranna Nixon RA. Neurofilaments and neurofilament proteins in health and disease. Cold Spring Harb Perspect Biol. 2017;9:a018309.

36. Nakamura R, Sone J, Atsuta N, Tohnai G, Watanabe H, Yokoi D, et al. Next-generation sequencing of 28 ALS-related genes in a Japanese ALS cohort. Neurobiol Aging. 2016;39(219):e1-8.

37. Nishiyama A, Niihori T, Warita H, Izumi R, Akiyama T, Kato M, et al. Comprehensive targeted next-generation sequencing in Japanese familial amyotrophic lateral sclerosis. Neurobiol Aging. 2017;53:194.e1-194.e8.

38. Morgan S, Shatunov A, Sproviero W, Jones AR, Shoai M, Hughes D, et al. A comprehensive analysis of rare genetic variation in amyotrophic lateral sclerosis in the UK. Brain. 2017;140:1611-8.

39. Maciel R, Correa R, Bosso Taniguchi J, Prufer Araujo I, Saporta MA. Human tridimensional neuronal cultures for phenotypic drug screening in inherited peripheral neuropathies. Clin Pharmacol Ther. 2020;107:1231-9.

40. Agrawal PB, Joshi M, Marinakis NS, Schmitz-Abe K, Ciarlini PD, Sargent $J C$, et al. Expanding the phenotype associated with the NEFL mutation: neuromuscular disease in a family with overlapping myopathic and neurogenic findings. JAMA Neurol. 2014;71:1413-20.

41. Lavedan C, Buchholtz S, Nussbaum RL, Albin RL, Polymeropoulos MH. A mutation in the human neurofilament M gene in Parkinson's disease that suggests a role for the cytoskeleton in neuronal degeneration. Neurosci Lett. 2002;322:57-61.

42. Wataya T, Nunomura A, Smith MA, Siedlak SL, Harris PL, Shimohama S, et al. High molecular weight neurofilament proteins are physiological substrates of adduction by the lipid peroxidation product hydroxynonenal. J Biol Chem. 2002;277:4644-8.

43. Pecoraro V, Mandrioli J, Carone C, Chiò A, Traynor BJ, Trenti T. The NGS technology for the identification of genes associated with the ALS. A systematic review. Eur J Clin Invest. 2020;50(5):e13228.

44. Liu ZJ, Lin HX, Wei Q, Zhang QJ, Chen CX, Tao QQ, Liu GL, Ni W, Gitler AD, Li HF, Wu ZY. Genetic spectrum and variability in Chinese patients with amyotrophic lateral sclerosis. Aging Dis. 2019;10(6):1199-206.

45. Chen W, Xie Y, Zheng M, Lin J, Huang P, Pei Z, Yao X. Clinical and genetic features of patients with amyotrophic lateral sclerosis in southern China. Eur J Neurol. 2020;27(6):1017-22.

\section{Publisher's Note}

Springer Nature remains neutral with regard to jurisdictional claims in published maps and institutional affiliations.

\footnotetext{
Ready to submit your research? Choose BMC and benefit from:

- fast, convenient online submission

- thorough peer review by experienced researchers in your field

- rapid publication on acceptance

- support for research data, including large and complex data types

- gold Open Access which fosters wider collaboration and increased citations

- maximum visibility for your research: over $100 \mathrm{M}$ website views per year
}

At BMC, research is always in progress.

Learn more biomedcentral.com/submissions 